\author{
Military Technical College \\ Kobry El-Kobbah, \\ Cairo, Egypt.
}

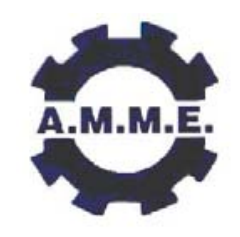
$13^{\text {th }}$ International Conference on Applied Mechanics and Mechanical Engineering.

\title{
NANO-REINFORCED COMPOSITE INTERFACES: OPPERTUNITIES AND CHALLENGES
}

\author{
MEGUID ${ }^{1}$ S.A.
}

\begin{abstract}
In the present paper, the delamination behaviour of nano-reinforced composite double cantilever beam is numerically studied. Specifically, it evaluates the influence of nanofillers on the reinforcement of adhesively bonded layer under mode-I fracture toughness using multiscale modelling. In this novel approach, we couple coarse-grain molecular dynamics with continuum mechanics. The molecular dynamics domain and the finite element domain are overlapped in a handshaking subdomain, The implementation of coarse-grain molecular dynamics radically reduces the size of the problem. An explicit algorithm coupling the two methodologies was developed and used to determine the energy release rates of cohesive cracks in adhesively bonded composite joints with varying amount of nano-reinforcement in the adhesive layer. Both the quality of the prediction of the multiscale model and the influence of the nanofillers are evaluated and discussed.
\end{abstract}

\section{KEY WORDS}

Nano-Reinforced Adhesive Layers; Carbon Nanotubes; Alumina Nanopowder; Multiscale Materials Modelling; Coarse-Grain Molecular Dynamics; Finite Element; Fracture Behaviour; Energy Release Rate.

1 Engineering Mechanics and Design Laboratory, Department of Mechanical and Industrial Engineering, University of Toronto, 5 King's College Road, Toronto, Ontario M5S 3 G8,

CANADA. Email: meguid@mie.utoronto.ca 


\section{INTRODUCTION}

Recent advances in airframe design led to the increasing use of advanced composites. In these composites, high strength adhesives are typically used to form the laminate structures using special processes such as autoclaving or sandwich bonding. The use of adhesive bonding as a joining method connecting different aerospace components has been shown to attain high structural efficiency, integrity and improved fatigue life. For example, in aircraft construction and repair, many structural components such as wing stiffeners, fuselage longerons and skin panels are adhesively bonded. The Boeing 747 has more than $60 \%$ of its wetted area constructed by means of adhesive bonding, while the Lockheed C-5A aircraft contains $35,000 \mathrm{ft}^{2}$ of bonded structures [1]. However, the mechanical properties and the stress concentration at the adhesive layers and their interfaces with the adherends have been one of the prime concerns in adhesive joints. Various techniques have been adapted to reduce this stress concentration and hence improve the loading bearing capacity. These include selection of the high-strength and high-toughness adhesive materials, dispersing fillers throughout the adhesives, altering and the adhesive and adherend geometry [2]. Reinforcing the epoxy adhesive by adding nanofillers is a new technique, which is receiving a great deal of attention [3-4]. The exceptional and superior physical and chemical properties of nanomaterials have significantly influenced materials design technology [5-6]. The study of the behaviour of these materials generally requires investigation at the atomic or molecular levels. Furthermore, in most applications, nanoscale materials are used in conjunction other components which are geometrically many orders larger, having different response times, and thus operating at very different length- and time- scales [7-9]. It is therefore our intention to develop a multiscale modelling technique to simulate fracture and delamination behaviour for nano-reinforced composite structures.

\section{FORMULATION OF PROBLEM}

Fig. 1 shows that the two substrates are joined together by the nano-reinforced epoxy adhesive layer and the computational domain is divided into two regions; namely, the molecular dynamics (MD) region and finite element region as show in Fig. 2. In the MD region, molecules or particles may be distributed either randomly or regularly. The kinetic energy $T$ and potential energy $V$ for the system with $N$ atoms are respectively

$$
\begin{gathered}
T=\frac{1}{2} \sum_{i=1}^{N} m_{i} v_{i}^{2} \\
V=\frac{1}{2} \sum_{i, j=1}^{N} V_{i j}+\sum_{i=1}^{N} V_{b i}-\sum \bar{F}_{i} r_{i}
\end{gathered}
$$

where the first term in $V$ is for the non-bonded interactions and the second for bonded interactions, while the last term represents the work done by external forces. The nonbonded interactions between atoms consist of repulsive and attractive forces, and are computed using the Lennard-Jones (LJ) potential on the basis of the neighbour list [1011].

Here we define the lower case subscripts ( $i$ and $j$ ) to denote atomic particles, and $r_{i}$ is the position vector of particle $i$, and using this notation: $r_{i j}=r_{j}-r_{i}$. The Hamiltonian energy of a system of molecules with $n$ particles is given by: 


$$
\begin{aligned}
H & =\sum_{i=1}^{N} P_{r_{i}} \dot{r}_{i}-L \\
& =\frac{1}{2} \sum_{i}^{N} \frac{P_{r_{i}}^{2}}{m_{i}}+\sum_{i=1}^{N} 4 u_{0}\left(\frac{d^{12}}{r_{i j}^{12}}-\frac{d^{6}}{r_{i j}^{6}}\right)+\sum_{i}^{N} \frac{1}{2} k_{i j}^{b}\left(r_{i j}-b_{i j}\right)^{2}-\sum_{i}^{N} \bar{F}_{i} r_{i}
\end{aligned}
$$

If the surface tensor and interaction in the system is harmonic, applying the Hamiltonian equations result in this following compact form

$$
[m] \frac{d^{2}}{d t^{2}}\{r\}=\left\{\left[F_{n b}\right]+\left[F_{b i}\right]+\{\bar{F}\}\right\}
$$

where $[\mathrm{m}]$ is the mass matrix, $\left[F_{n b}\right]$ and $\left[F_{b i}\right]$ are the non-bonded and bonded interaction forces respectively, and $\{\bar{F}\}$ is the external load vector. Given the initial positions and external forces of the system, integration of the above equations yields the total trajectory of the system. These results will be introduced to the finite element analysis as boundary conditions. The initial conditions are estimated using a Monte Carlo technique, while the initial velocity components $v_{i}$, where $i=1 \ldots 3 N$, are generated based on the Maxwellian distribution at a given absolute temperature $T$ [12].

Based on statistical mechanics, the local stress tensor $\sigma_{i j}$ can be obtained by imposing conservation of linear momentum $p(r)[13]$ :

$$
\frac{d}{d t} p(r)=-\frac{\partial}{\partial x_{j}} \sigma_{i j}
$$

where the momentum is defined in terms of the individual particle's $(\alpha)$ momenta and position, $p^{\alpha}$ and $r_{\alpha}$. Using Newton's second law, one obtains the stress tensor

$$
\sigma_{i j}(r)=\sum_{\alpha} m^{\alpha} v_{i}^{\alpha} v_{j}^{\alpha} e^{i k r_{\alpha}}+\frac{1}{2} \sum_{\alpha \neq \beta}\left(\frac{\partial V}{\partial r_{\alpha \beta}} r_{\alpha \beta}\right) \frac{r_{\alpha \beta} r_{\alpha \beta}}{r_{\alpha \beta}^{2}} \frac{e^{i k r_{\alpha}}-e^{i k r_{\beta}}}{i k r_{\alpha \beta}}
$$

Here we define a coarse-grained average stress by integrating the local stress over an appropriate averaging volume $\Omega$ around particle $\alpha$., taking the continuum limit of the above, i.e., $\mathrm{k} \rightarrow 0$, we obtain

$$
\bar{\sigma}_{i j}(r)=\frac{1}{\Omega}\left\{\sum_{\alpha} m^{\alpha} v_{i}^{\alpha} v_{j}^{\alpha}-\frac{1}{2} \sum_{\alpha \neq \beta} \frac{r_{\alpha \beta}^{i} r_{\alpha \beta}^{j}}{\left|r_{\alpha \beta}\right|} \frac{\partial V}{\partial r_{\alpha \beta}}\right\}
$$

In terms of finite element modelling, it straight forward to obtain the total potential energy of the system, which would lead us to

$$
\sum_{e=1} \frac{\partial \Pi^{e}}{\partial\{d\}}=\sum_{e=1}\left(\left[K^{e}\right]\{d\}-\{F\}-\{\Phi\}\right)=0
$$

where $\Pi$ is system potential energy and $d$ is displacement, $\left[K^{\mathrm{e}}\right],\{F\}$ and $\{\Phi\}$ are the element stiffness matrix, body force vector and surface traction vector, respectively. 
The FE mesh is composed of 8 noded solid elements with uniform thickness. The left edge in the FE regime is subjected to hinge forces in opposite directions, and the right edge in FE regime is constrained in the length and thickness directions. Near the crack region, the adhesive and handshaking substrate areas are modelled using molecular dynamics. Further details can be found in our published work.

\section{RESULTS AND DISCUSSIONS}

Simulations for the mode I opening of a double cantilever beam were carried out to obtain relations between load and crack opening displacement, load and crack length, and also between interfacial strain energy release rate $G$. The cantilever beam model consists of two rectangular, uniform thickness, unidirectional carbon fibre laminated composites pieces bonded together by epoxy adhesive. A pre-crack in the adhesive layer, with a length of $50 \mathrm{~mm}$, is used in the model to serve as a delamination initiator. Opening forces are applied to the DCB at the upper left or lower left side. Opening displacements and delamination length were then computed and compared with experimental results. The solution procedure involves application of incremental loads with iterative loads. The system energy is simulated and shown in Fig. 3. The epoxy adhesive with dispersed $5 \%$ nanopowder presents the highest energy as a function of time, while the epoxy adhesive with dispersed $5 \%$ nanotubes shows moderately high energy as a function of time, and the pure epoxy shows the lowest energy as function of time. The difference in the energy is a measure of the elastic and plastic energy stored by the system. The stored energy tends to be lower for the pure epoxy which has less cohesive energy and therefore lower resistance to fracture. In contrast, the system with a suitable amount of nanoparticles reinforcement tends to show higher cohesive energy and therefore higher resistance to fracture. Reported experimental data $[4,14]$ suggests that approximately $5 \mathrm{wt} \%$ provides the greatest reinforcement for the systems considered.

The relations between loading and delamination (or crack) length are shown in Figs. 4 (left column) for CF-CF double composite cantilever beams. Simulations are carried out by using pure epoxy adhesive, epoxy adhesive with dispersed nanotubes (5\%) and epoxy adhesive with dispersed nanopowder (5\%). When the load increases, the delamination length also increases. Simulation results show excellent agreement with the experimental results. The nano-reinforced adhesives display better adhesion properties compared with pure epoxy, and the epoxy adhesive with nanopowder reinforcement performs better than that with nanotube reinforcement.

The relation between energy release rates of DCB specimens and crack-length were computed. The results are presented in Fig. 4. Results are corresponding to pure epoxy adhesive, epoxy adhesive with nanotubes (5\%) and epoxy adhesive with nanopowder $(5 \%)$. They show that the predicted energy release rates are dependent on the range of crack lengths. The nano-reinforced adhesives display better adhesion properties compared with pure epoxy, and the epoxy adhesive with nanopowder reinforcement performs better than that with nanotube reinforcement. These can be shown by comparing the energy release rates at a typical crack length. For example, when total crack length is $70 \mathrm{~mm}$, pure epoxy bonded DCB gives $G=400 \mathrm{~J} / \mathrm{m}^{2}$, the nanotube reinforced DCB has $467 \mathrm{~J} / \mathrm{m}^{2}$, the nanopowder reinforced DCB has $G=627 \mathrm{~J} / \mathrm{m}^{2}$. As expected, the simulation results generally predict slight higher values as compared to 
the experimental results. This over prediction may be primarily attributed to the ideal nature of the material in the MD simulation. Additionally, the calculation method of $G$ differs for the simulation and the experiment.

\section{CONCLUSIONS}

In this paper, we have presented a novel efficient multiscale modelling scheme for the fracture analysis of a nano-reinforced epoxy adhesive layer, and applied it to the delamination of double cantilever beams with macroscopic dimensions. The model is based on a hybrid strategy and couples the macroscale-nanoscale fracture processes in which the continuum based finite element computations of the stress and displacement fields are integrated with the local nanoscale atomistic dynamics. The nanoscale molecular dynamics computations employed coarse-grained beads which significantly reduced computational time and made the simulation viable. The transition from the macroscale to the nanoscale was achieved via the introduction of an intermediate handshaking mesoscale zone with continuum-based stress and displacement fields. The nanoscale computations then provided the critical crack increments and the energy release rate of the crack propagation. The model has predicted the relations of loading as a function of delamination increments, and energy release rates as a function of crack length and nanoparticle weight percentage dispersed in the epoxy adhesive.

\section{REFERENCES}

1. Forte M.S., Whitney J.M. and Schoeppner G.A., The influence of adhesive reinforcement on the Mode I fracture toughness of a bonded joint. Composites Science and Technology, 2004; 60:2389-2405.

2. Boss J.N., Ganesh V.K. and Lim C.T., Modulus grading versus geometrical grading of composite adherends in single-lap bonded joints, Composite Structures, 2003; 62:113-121.

3. Naganuma T. and Kagawa Y., Effect of particle size on the optically transparent nano meter-order glass particle-dispersed epoxy matrix composites, Composites Science and Technology, 2002, 62(9): 1187-1189.

4. Meguid S.A., Sun $Y$, On the tensile and shear strength of nano-reinforced composite interfaces, Materials \& Design, 2004, 25: 289-296.

5. Qian D., Wagner G.J., and Liu W.K., Mechanics of carbon nanotubes, Applied Mechanics Review, 2002, 55(6), 495-533.

6. Lurie, S.A., Belov P.A., Tuchkova N.P., The application of the multiscale models for description of the dispersed composites, Composites: 2005, Part A 36: 145-152.

7. Park, H.S., Karpov E.G. and Liu W.K., A temperature equation for coupled atomistic/continuum simulations, Computer Method in Applied Mechanics and Engineering, 2004; 193:1713-1732.

8. Abrams C.F. and Kremer K., Combined coarse-grained and atomistic simulation of liquid bisphenol A-Polycarbonate: Liquid packing and intramolecular structure, Macromolecules, 2003, 36:260-267.

9. Shilkrot L.E, Curtin W. A. and Miller R.E., A coupled Atomistic/continuum model of defects in solids, Journal of the Mechanics and Physics of Solids, 2002; 50:2085-2106. 
10. Kremer K. and Grest G.S., Chapter 4, Monte Carlo and molecular dynamics simulation in polymer science, edited by Binder K., Oxford University Press, New York, 1995; 194-271.

11. Odegard G.M., Gates T.S., Wise K.E., Park C., Siochi E J., Constitutive modelling of nanotubes-reinforced polymer composites, Composites Science and Technology, 2003; 63(11), 1671-1687.

12. Spoel D. V. D., Buuren A.R., Apol E., Meulenhoff P.J., Tieleman D.P., Sijbers A.L.T.M., Hess B., Feenstra K.A., Lindahl E., Drunen R. V. and Berendsen H.J.C., Gromacs user manual version 3.1.1, Nijenborgh 4, 9747 AG Groningen, The Nethlelands, Internet:www.gromacs.org, 2002.

13. Cormier J., Rickman J.M. and Delph T.J., Stress calculation in atomistic simulations of perfect and imperfect solids, Journal of Applied Physics, 2001; 89(1):9-104.

14. Sun Y., Meguid S.A., Liew K.M., Ong L.S, Design and development of new nano-reinforced bonds and interfaces, Proc. Nanotech 2004, Mar. 8-11, 2004, Boston, USA, pp.126-129. 


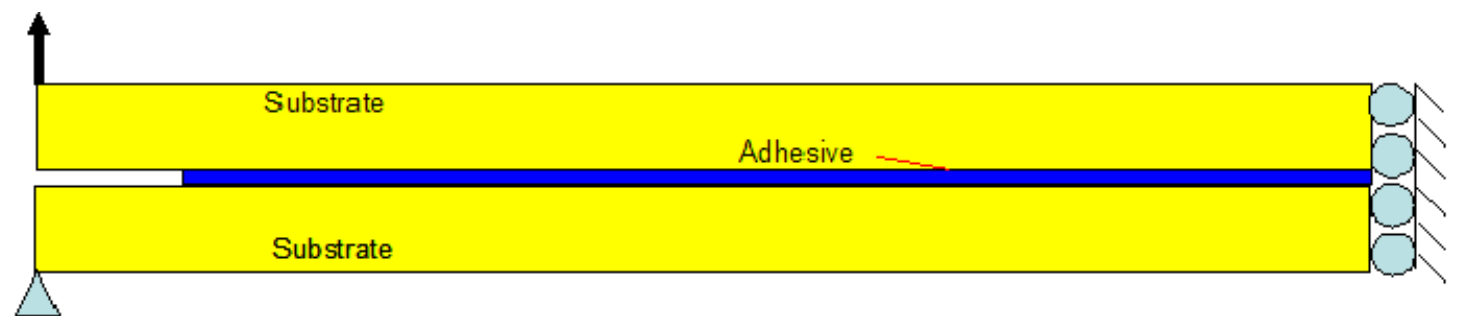

Fig. 1. Schematic of the DCB specimen consists of substrates and an adhesive layer.

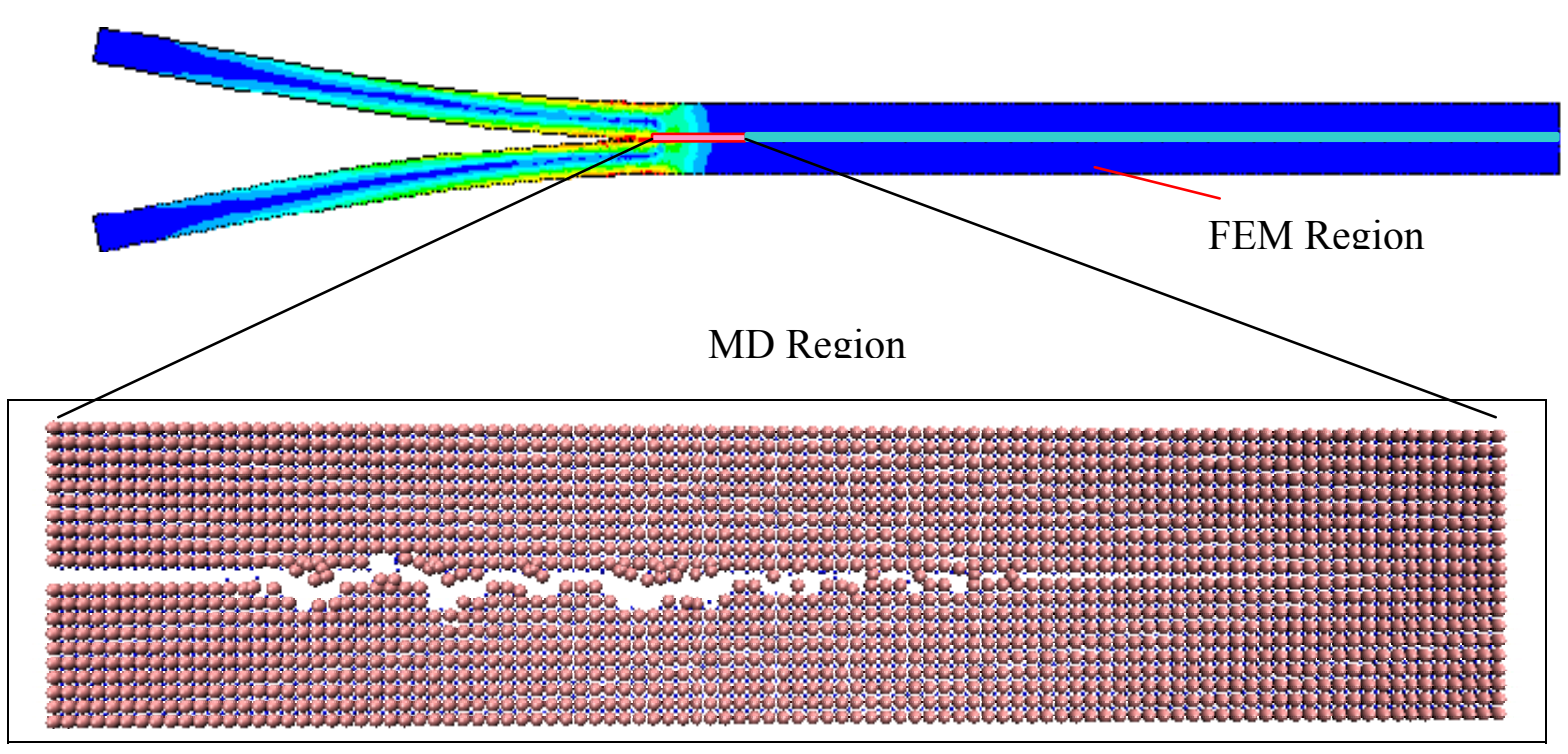

Fig. 2 Regions of finite element (FE) region and molecular dynamics (MD)

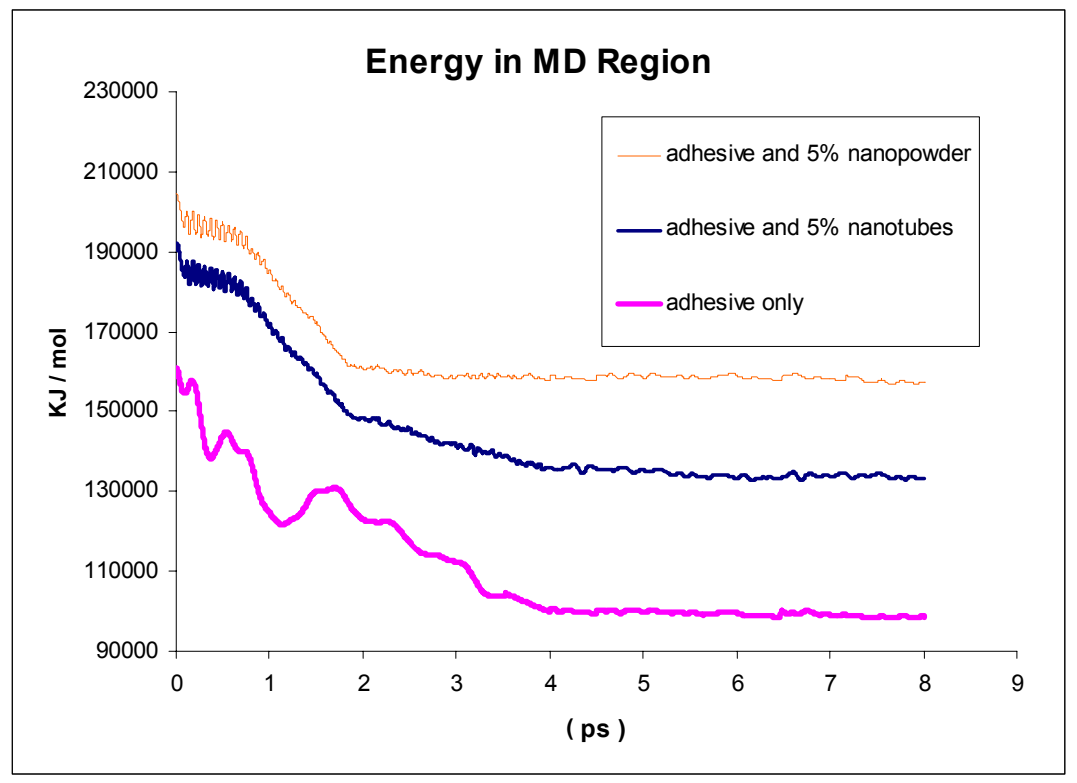

Fig. 3 Energy of the MD region 


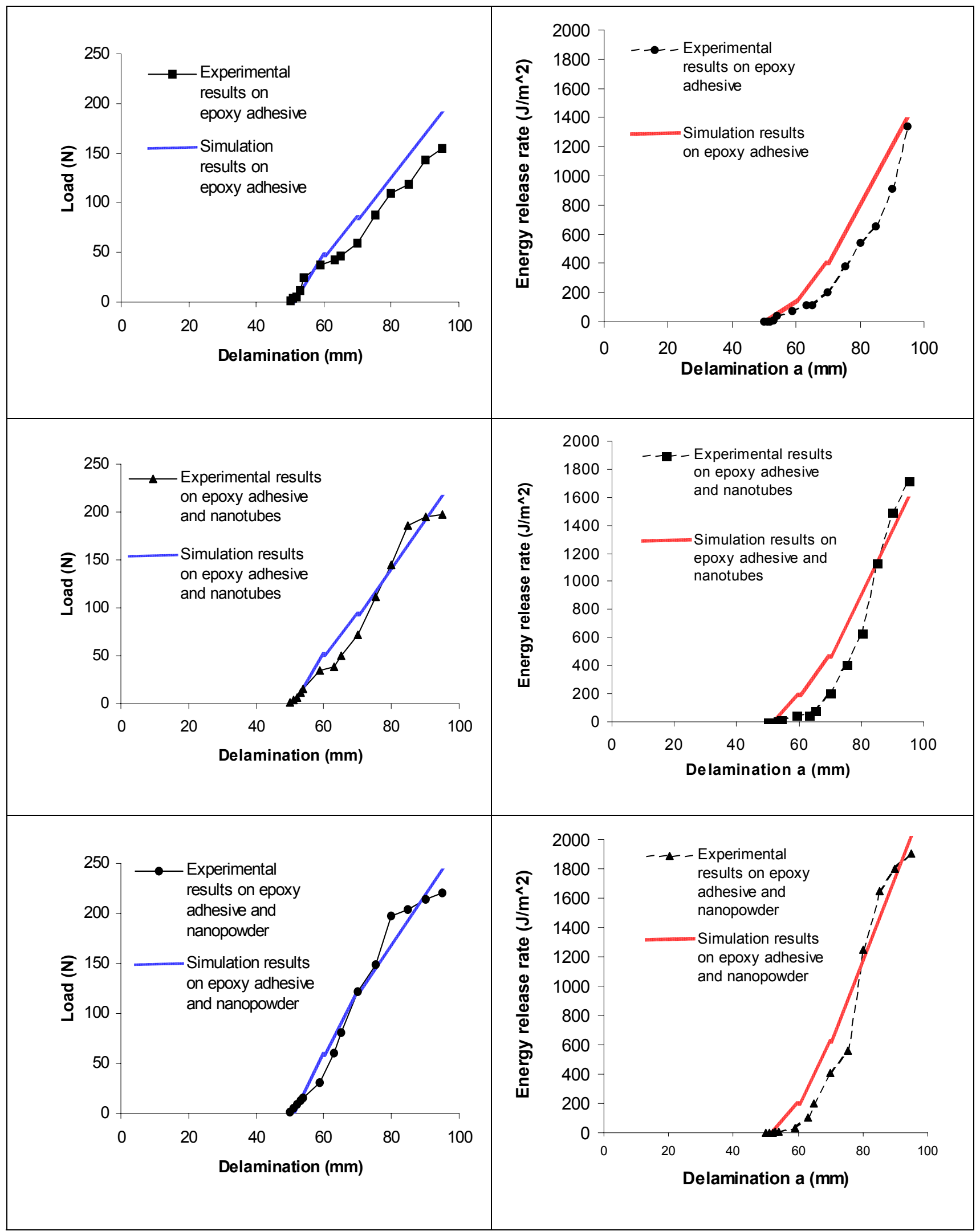

Fig. 4. Load /energy release rate vs. delamination for CF-CF substrates bonded by (Top) pure epoxy; (Mid) nanotube reinforced epoxy adhesive, and (Bottom) nanopowder reinforced epoxy adhesive. 


\title{
BIOGRAPHICAL SKETCH
}

\author{
Shaker A. Meguid
}

PhD, PE, CEng, FIMechE, MASME, LTM-AIAA
Professor and Director, Engineering Mechanics and Aerospace Design Laboratory
Department of Mechanical and Industrial Engineering University of Toronto
CANADA

Editor-in-Chief of Int. J. Mechanics and Materials in Design
Former Head, Aerospace Engineering Division
Nanyang Technological University
50 Nanyang Avenue
SINGAPORE
Former Director, Aerospace Centre of Excellence
Nanyang Technological University
50 Nanyang Avenue
SINGAPORE

Approved Consultant for

United Nations Industrial Development Agency (UNIDA), 2006-Current

\begin{abstract}
Dr. Meguid received his B.Sc. in 1969 and his M.Sc. in 1973 from Cairo University. His M.Sc. research work on constitutive modelling of solids was conducted at the Technical University of Denmark (1971-1972). He obtained his Ph.D. in Applied Mechanics in 1975 from the University of Manchester Institute of Science and Technology, England. He then joined the Engineering Science Department of Oxford University in 1976 as a Postdoctoral Fellow to work on the dynamic behaviour of rate sensitive materials. In view of his excellent research and teaching skills, he was invited to tutor for Magdallen and Pembroke colleges at Oxford University.
\end{abstract}

In 1980, he joined Cranfield University (England) as a Lecturer and, in view of his significant contribution to the Applied Mechanics Division, he became a Senior Lecturer in 1983. Dr. Meguid was invited by the late Professor Tabarrok to join the Department of Mechanical Engineering of the University of Toronto in July 1988 as a Senior Associate Professor. In July 1990 , he was promoted to the rank of full Professor.

In June 2004, the Dean of Engineering of Nanyang Technological University (NTU), Singapore, invited Professor Meguid to start a new aerospace division. He felt it was a great opportunity and a challenge. Following the approval of U of T, he joined NTU in September 2004. As the Founding Head, he designed and conceived the entire foundation of the new division and degree program. As custodian of the program, he devised and implemented 
highly innovative curricula that ensured active and interactive learning. In addition, he was instrumental in the design of a new aerospace building with over 17 highly specialised stateof-the-art laboratories costing the equivalent of $\mathrm{CDN} \$ 52$ millions. The building, which contains a wind tunnel and a flight simulator, also accommodates a skyhawk $\mathrm{A} 4, \mathrm{H} 1 \mathrm{UH}$ helicopter and numerous instrumented artefacts. In view of his passion and commitment to his scholarly work, he continued his research in five main areas: (i) Transient Response of Thermo-Electro-Mechanically Coupled Smart Functionally Graded Structures, funded by NSERC from 2002-2007. Although his funds were frozen in years 2005 and 2006, as per NSERC rules, he continued his research uninterrupted. The remainder of his funds was kindly re-instated on his return in June 2007, (ii) Nanomechanics of Single and Multi-Walled Carbon Nanotubes, NTU (Matching Fund), (iii) Modelling and Characterisation of Cellular Materials, Defence Science Organisation National Labs (DSO), Singapore, (iv) The Transient Response of a Bird Striking a Fan Blade, DSO-NTU (Matching Fund), and (v) Wing Morphing using Shape Memory Alloys funded by DSO. In addition, he continued the supervision of his postgraduate students by regularly visiting $\mathrm{U}$ of $\mathrm{T}$, through videoconferencing, email and telephone. During his leave in Singapore, he succeeded in graduating $3 \mathrm{PhD}$ and 2 masters students in Toronto, and attracting three new students to join his team. He is currently negotiating the transfer of the projects from NTU Singapore to the University of Toronto.

Dr. Meguid is a very creative researcher with immense vision. He is capable of launching major projects and seeing them to their suocessful conclusion. He founded the Engineering Mechanics and Design Laboratory (EMDL) at the University of Toronto (U of T) in 1990. Within five years of arrival, Meguid built it up into a vibrant facility with 14 researchers and an a large operating budget. In addition to being well funded and supported by industry and government agencies, his research and development work was supported by three centres of excellence: MRCO, OCMR and IRIS. Dr. Meguid conducted numerous industrial short courses on fracture and fatigue of metals, shot peening to combat fatigue, and finite element modelling. His succeeded in attracting aocumulative career research grants/contracts/consulting in excess of 20 million dollars.

His research activities, which span over thirty years, have contributed significantly to the areas of nano and micromechanics, variational inequalities, contact finite element analysis, micromechanics of coupled field problems such as smart structures, and fracture mechanics of interfaces. He has published numerous papers in leading scientific journals with high impact factors, including The Proceedings of the Royal Society of London, ASME Journal of Applied Mechanics, International Journal of Numerical Methods in Engineering, International Journal of Solids and Structures, Journal of Mechanics \& Physics of Solids, among others. He has contributed to numerous symposia in Europe and North America as a keynote speaker in these topics. Professor Meguid is the Editor of eight international procedings encompassing design, finite element modelling, fatigue and fracture of engineering materials. $\mathrm{He}$ is the author of two books; titled: (i) Integrated Computer Aided Design of Mechanical Systems (1987), and (ii) Engineering Fracture Mechanics (1989), which received excellent reviews. 
The review of his Fracture Mechanics book stated, "if one were to own a single fracture mechanics book, this will be a good choice". The review of his Integrated CAD book stated under "Modest Titles Hides a Jewel-This book will without a doubt become an essential reading material for modern engineers".

$\mathrm{He}$ is an active member of a number of International Scientific Committees and Standards Boards, Departmental Committees, former President of the British Impact Treatment Association; Member of SAE Fatigue Enhancement Division, and the Wave Propagation Committee of ASME. He is the founding Editor-in-Chief of the International Journal of Mechanics and Materials in Design, Past Associate Editor of ASME Journal of Engineering Materials and Technology and a member of the editorial board of a number of journals, including: Finite Elements in Analysis and Design, Damage Mechanics and Journal of Materials Processing Technology. He is approached regularly by the members of the media for clarification of engineering issues concerning the public and/or of public interest.

Dr. Meguid is an approved consultant for the United Nations Industrial Development Agency, a Professional Engineer (PE) in the province of Ontario, a Chartered Mechanical Engineer (CEng) and a Fellow (FIMechE) of the Institution of Mechanical Engineers of Great Britain and a member of the American Society of Mechanical Engineers (MASME), Life Time Member of American Institute of Aeronautics and Astronautics LTM-AIAA, among others. $\mathrm{He}$ is also a scientific advisor to the University of Porto (Portugal) and an external examiner to Dublin Institute of Technology, and a number of universities in Asia. Dr. Meguid is a visiting Professor at the Department of Mechanical and Manufacturing Engineering of The Nottingham Trent University (TNTU), England, and the School of Mechanical and Aerospace Engineering (MAE) of Nanyang Technological University (NTU), Singapore, Aerospace Department, Tongji University, Shanghai, China. As a result of his association with TNTU, a strategic collaborative alliance with $\mathrm{U}$ of $\mathrm{T}$ was formed in 1999. And, as a result of his association with NTU, an additional strategic alliance with the Republic of Singapore Air Force (RSAF) and MPE was developed in 2004. Some eleven research projects in six disciplines in aerospace/mechanical engineering are currently being considered for funding by RSAF in Nanyang Technological University.

Dr. Meguid held the Wallace G. Chalmers Chair of Engineering Design for 5 years. His department nominated him for the CANCAM Applied Mechanics Award in 1998. In recognition of his contribution to knowledge, his international reputation, close collaboration with industry, commitment to the engineering profession and overall teaching, research and administrative performance at the University of Toronto, Meguid achieved and maintained the highest yearly ranking possible (outstanding) amongst the senior faculty for some 10 years. 